\title{
RARE-EARTH SYSTEMATICS IN Ap AND Am STELLAR SPECTRA: ARE NEW THEORETICAL DEVELOPMENTS REQUIRED?
}

\author{
Charles R. Cowley \\ University of Michigan, and Dominion Astrophysical Observatory, Victoria, B.C., Canada
}

ABSTRACT

The very large overabundances of rare earth elements found in Ap stars $\left(>10^{3}-10^{5}\right)$ usually arise in cases where the observable second spectra does not represent the dominant ionization stage. The extreme overabundance factors are highly correlated with effective temperature in a way that is regarded with suspicion. In cool Ap stars, where one does observe the dominant ionization state, more modest overabundances $\left(\sim 10^{2}\right)$ occur. The typical cool Ap and Am stars must have comparable abundances of the lighter lanthanides. It is argued that the sharp rise in typical Ap star abundances that parallels the predicted degree of ionization may be due to a breakdown in the Saha Equation or possibly to the existence of a cool upper photosphere caused by line blanketing in the hotter Ap stars.

Attention is called to the observational fact that the cool Am stars have deeper line cores than chemically normal main sequence stars or cool Ap stars. These line cores resemble those of more luminous stars, a fact which comports well with some (though not all) low dispersion similarities between Am stars and evolved objects. While various studies (e.g. by Conti and Van't Veer Menneret) have shown that reduced gravity models cannot remove the abundance anomalies in the Am stars, some modification of the traditional models and/or line formation theory is indicated in order to explain the observations.

I. DOES THE SAHA EQUATION BREAK DOWN IN HOT AP STARS?

It has been several years now, since the methods ard results of stellar abundance workers have come under severe criticism (cf. Worrell and Wilson 1972). Even the great non-1ocal 
thermodynamic equilibrium (LTE) controversy is muted. The substantial successes won by the non-LTE techniques in treating the spectra of the hottest stars as well as selected features in cooler stars has apparently slaked the appetites of the stellar atmosphere theoreticians for criticism of the older LTE methods.

Yet, we must always be on our guard. One of the strongest arguments for the LTE methods was always that they were able to account in a self consistent way for many observations at the level of accuracy applicable at that time. Such an argument can not necessarily be expected to hold as new or more extensive observations become available. The purpose of the present paper is to discuss some generalities which have emerged as a result of our high dispersion surveys (Cowley 1979) of B, A and F stars both normal and peculiar (see Deutsch 1956). The spectra were obtained at $2 \AA / \mathrm{mm}$ at the 48 -inch Coude of the Dominion Astrophysical Observatory (DAO). It is our present feeling, that the general relations which we will discuss require a modification in the techniques traditionally used for the analysis of such spectra, although the nature of the modifications required is not yet clear. Certain results point to a breakdown of the ionization equation, while others suggest inadequacies in current stellar models.

Consider, for example, the differences in abundances of the lanthanide rare earths obtained in magnetic Ap stars and in metallic line A stars. It is generally held that the overabundance factors for the lanthanides are one-to-two orders of magnitude larger in the first group of stars than in the second. This result may be traced directly to the fact that the lanthanides can be identified in magnetic Ap stars whose effective temperatures imply a high degree of double ionization. For example, at $10,000^{\circ} \mathrm{K}$, less than $1 \%$ of the cerium is in the easily observable singly ionized state at a typical point in an A star atmosphere $\left(\log \mathrm{P}_{\mathrm{e}} \approx 2.0\right)$.

We are, therefore, obtaining abundances from the non-dominant stage of ionization, and relying on our models and (usually LTE) diagnostic techniques to supply the large correction factors accurately. Extensive spectroscopic analysis of the dominant, second stage of ionization is only now becoming possible, (Aikman et al. 1979) and transition probabilities are not yet available. For the most part, the stronger lines of the third spectra (2nd ionization stage) of the lanthanides fall outside of the region generally included in our own surveys. However, enough lines do occur in the region ca. $3700-4700 \AA$ to allow unambiguous identifications in a few instances.

Generally, whenever we find the third spectra of the lanthanides, the second spectra are still present and relatively strong, even when we examine stellar spectra originating from photospheres where $10^{-3}-10^{-4}$ of the lanthanides are supposedly singly ionized (cf.HD 200311 Adelman 1974; HD 187473, Hensberge et al. 1980). There is something very strange about this behavior. Let us compare it with the observations of the first and second spectra of iron 
group elements. Between spectral types FO and AO, Ti I, Cr I, Fe I, etc. weaken markedly and steadily. A similar behavior is expected from lanthanide spectra between types $A O$ and B7-B8 $\left(\mathrm{T}_{\mathrm{e}} \sim 13,000^{\circ} \mathrm{K}\right)$, where the change in ionization is similar. We do observe a weakening of most lines but it is straightforward to ascribe this to the increase in the Paschen continuous opacity. The crucial point is that a weakening of the second spectra of the lanthanides relative to the third is not obvious.

It is well known that if we observe lanthanide spectra at all among stars with effective temperatures greater than $10,000^{\circ} \mathrm{K}$, we are looking at stars with abundance anomalies, and such objects are a very heterogeneous group. We have not yet obtained a large sample of such stars with sharp-lined spectra, so it may still be possible to explain the lack of a general trend in terms of the abundance fluctuations from star to star, but I do not think so.

The time is now ripe for theoretical efforts which might explain the abundance anomalies which mimic so closely ionization corrections. Clearly, if the explanation is to be found in the analyses, there must be some reason why the degree of ionization in the regions of the stellar photosphere where the lanthanide lines are formed is lower than our "standard" calculations tell us. There are several possibilities:

1. U.V. flux redistribution may make the color temperatures (eg. Strbmgren colors) too high.

2. Line blanketing may create an anomalously cool outer photospheric boundary in which the rare earths are predominently first ionized.

3. The LTE Saha algorithm is predicting too high a degree of ionization. The first two effects are currently under study (Leckrone 1980, Kurucz 1979), a1though not in precisely the present context. Since they work qualitatively in the desired direction, the question is whether these mechanisms can account for the observations quantitatively. Useful results should be available within the next year or so. A realistic non LTE analysis of lanthanide spectra may be somewhat further off.

II. RARE EARTHS IN THE COOLER AP and Am STARS

Several of the cooler Ap stars, for which the dominant ionization stage is the second, have been studied for abundances (see the review by Hack 1976). These studies appear to show overabundance factors 10-100 times those of Am stars with similar temperatures, and indicate that the difference between typical Am and Ap abundances is not only due to perverse ionization corrections. The results, we believe are somehow in error, and that the lanthanide abundances in the coolest Ap and Am stars are closely comparable.

We base this assertion upon observational material that is unquestionably equal to or superior to that used in earlier abundance studies where the more commonly accepted ideas 
about abundance excesses in these stars originates.

Table 1 gives the results of our wavelength coincidence statistics for La II and Ce II in several cool Am and Ap stars. Effective temperatures for these stars are very difficult to determine accurately, but this is not of great importance because all of these stars are cool enough that the singly ionized state is the dominant one; ionization corrections will not be significant. Rough temperature estimates from Cowley and Aikman (1980) are given in the table.

Table 1. Coincidence statistics for La II and Ce II in cool Ap and Am stars

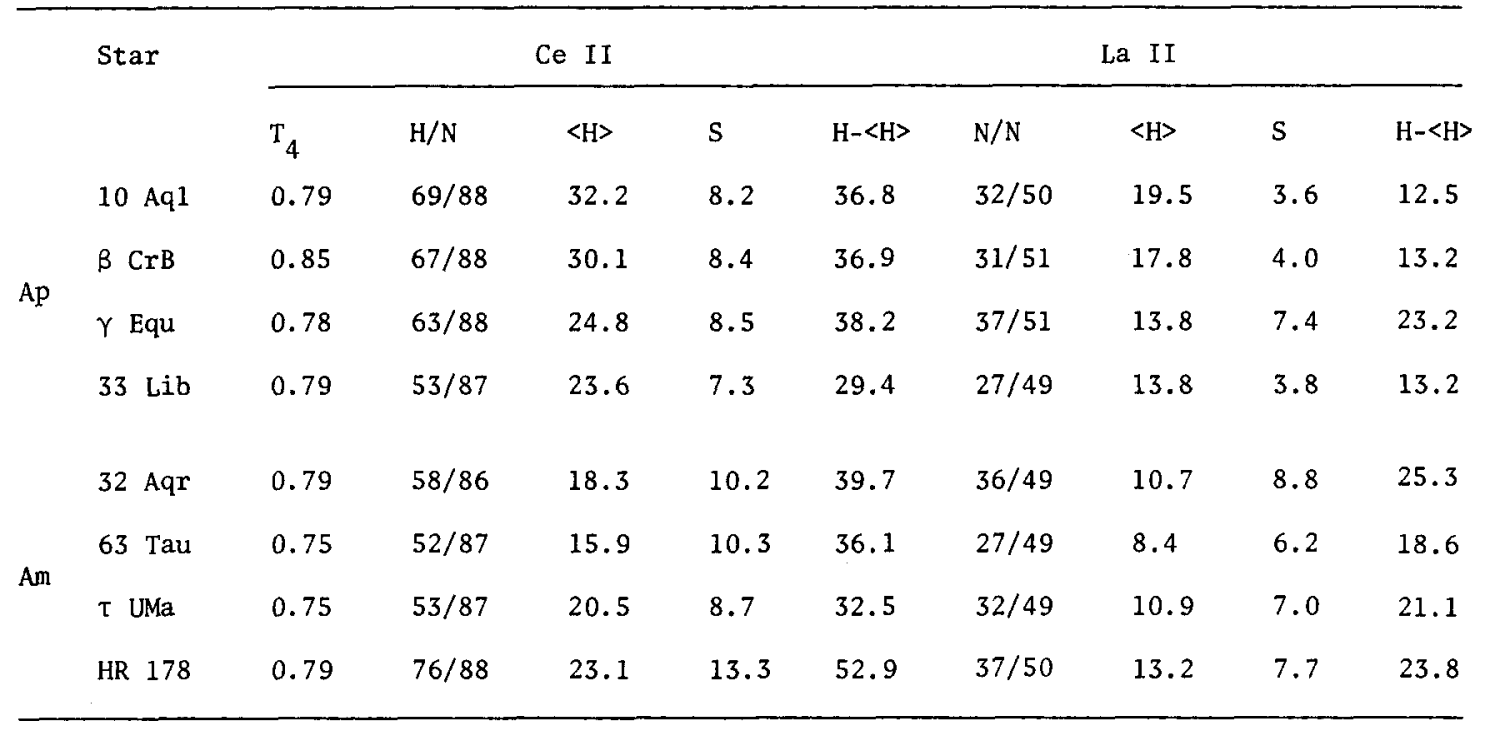

The column headed H/N gives the number of coincidences or "hits" $\mathrm{H}$ out of $\mathrm{N}$ lines sought, using our standard tolerance of $\pm 0.06 \AA$. The average number of coincidences on $N$ nonsense wavelengths is given under the column headed $\langle\mathrm{H}\rangle$, while $\mathrm{S}$ is the parameter describing the statistical significance, in standard deviations, of the $\mathrm{H}$ coincidences. A detailed description of these parameters is given by Hartoog et al. (1973). Since $\langle\mathrm{H}\rangle$ coincidences would occur even if La II and Ce II were not present (provided the line densities remained the same!), it is useful to examine the final column containing the "excess coincidences."

These figures show that there are just about the same number of faint La II and Ce II lines in the Am as Ap stars. The very sharp-1ined Am spectrum of HR 178 has an extraordinary number of $\mathrm{Ce}$ II coincidences. These numbers of coincidences are a good measure of the relative strengths of the fainter lines of the respective atomic spectra. Since faint lines are most sensitive to abundances, we conclude that the abundances of cerium and lanthanum in these cool Ap and Am stars are comparable.

Traditional abundance determinations are based on equivalent widths. These tell exactly the same story, which we illustrate in Figures 1-4 with $\lambda 4364$ which is a blend of Ce II and 


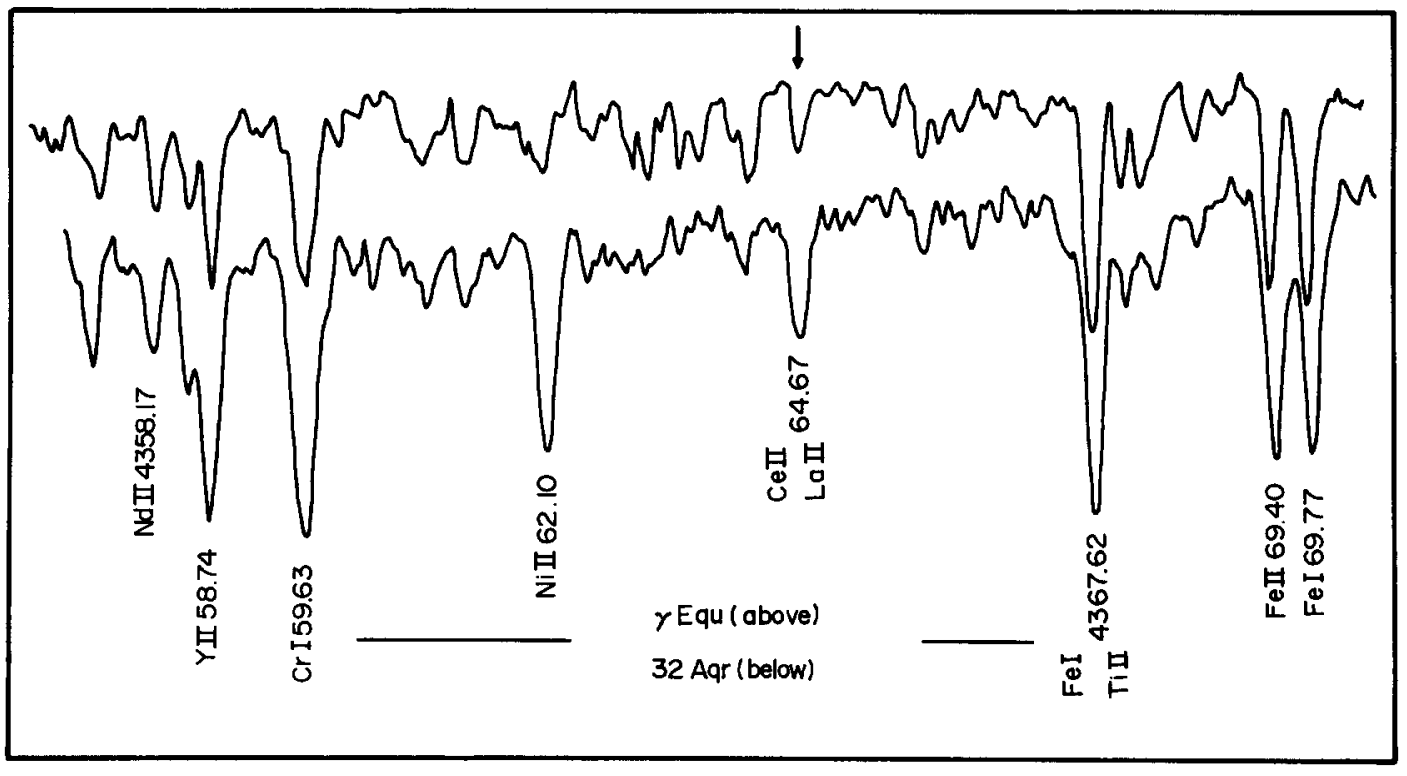

Figure 1. Direct intensity tracings of the region near $\lambda 4363$ of the Ap star $\gamma$ Equ and the Am star $32 \mathrm{Aqr}$. Zero intensity is at the position of the light horizontal line and the base of the figure for $\gamma$ Equ and 32 Aqr respectively. The arrow points to a blend of Ce II and La II near $\lambda 4364.67$. Nd II $\lambda 4358.17$ is also illustrated. These lines are of comparable intensities in the two spectra. The general level of ionization which may be inferred from the ratio $\mathrm{Fe}$ II $\lambda 4369.40 / \mathrm{Fe}$ I $\lambda 4369.77$, is similar in both stars. Note the great strength of $\mathrm{Ni}$ II $\lambda 4362.10$ in $32 \mathrm{Aqr}$. Strong Ni II and Y II are generally typical of Am stars but not necessarily of magnetic Ap stars. See the remaining figures.

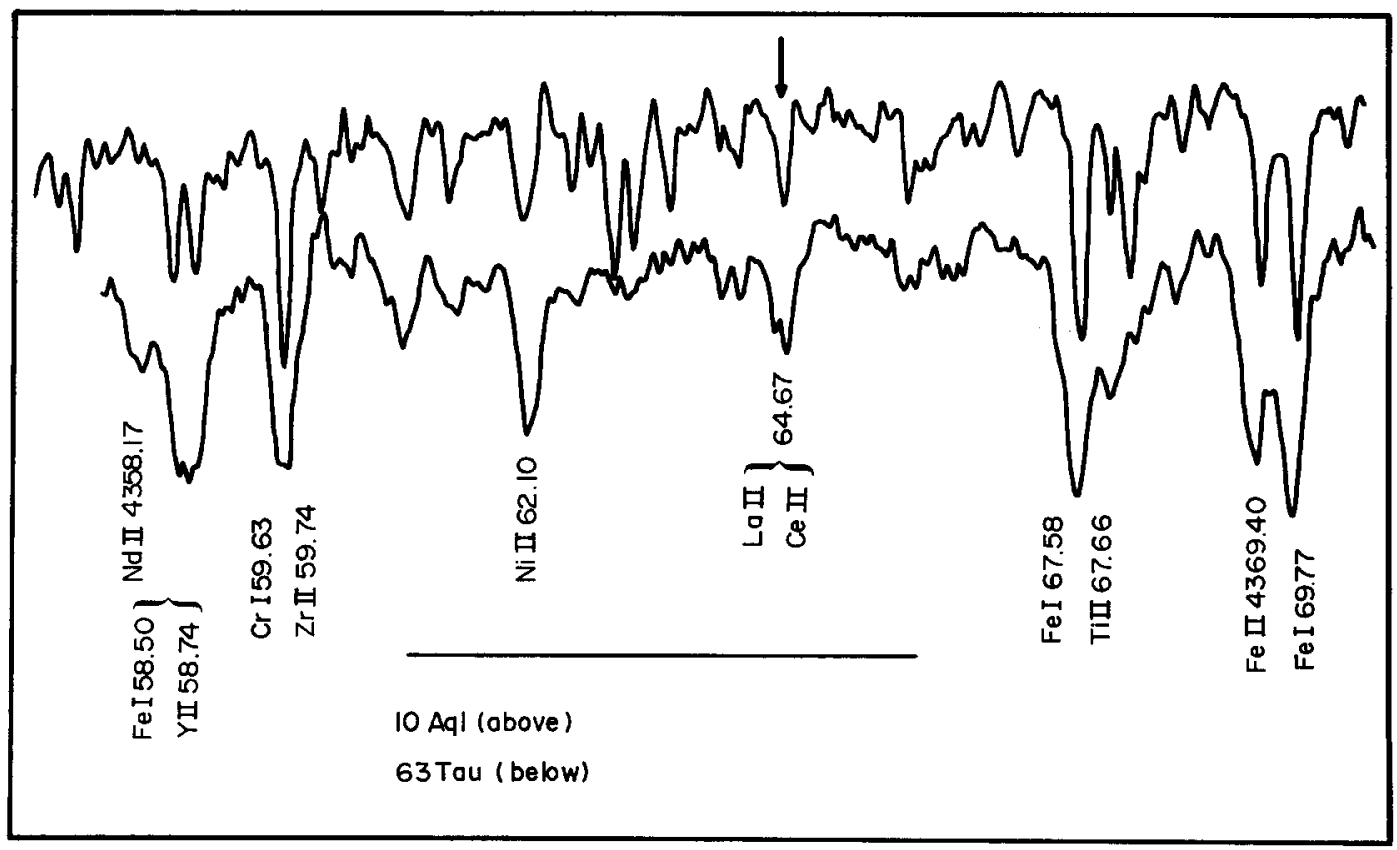

Figure 2. Same format as Figure 1 for the Ap star $10 \mathrm{Aql}$ and the Am star 63 Tau. Note the absence of Nd II $\lambda 4358.17$ in $10 \mathrm{Aql}$. 


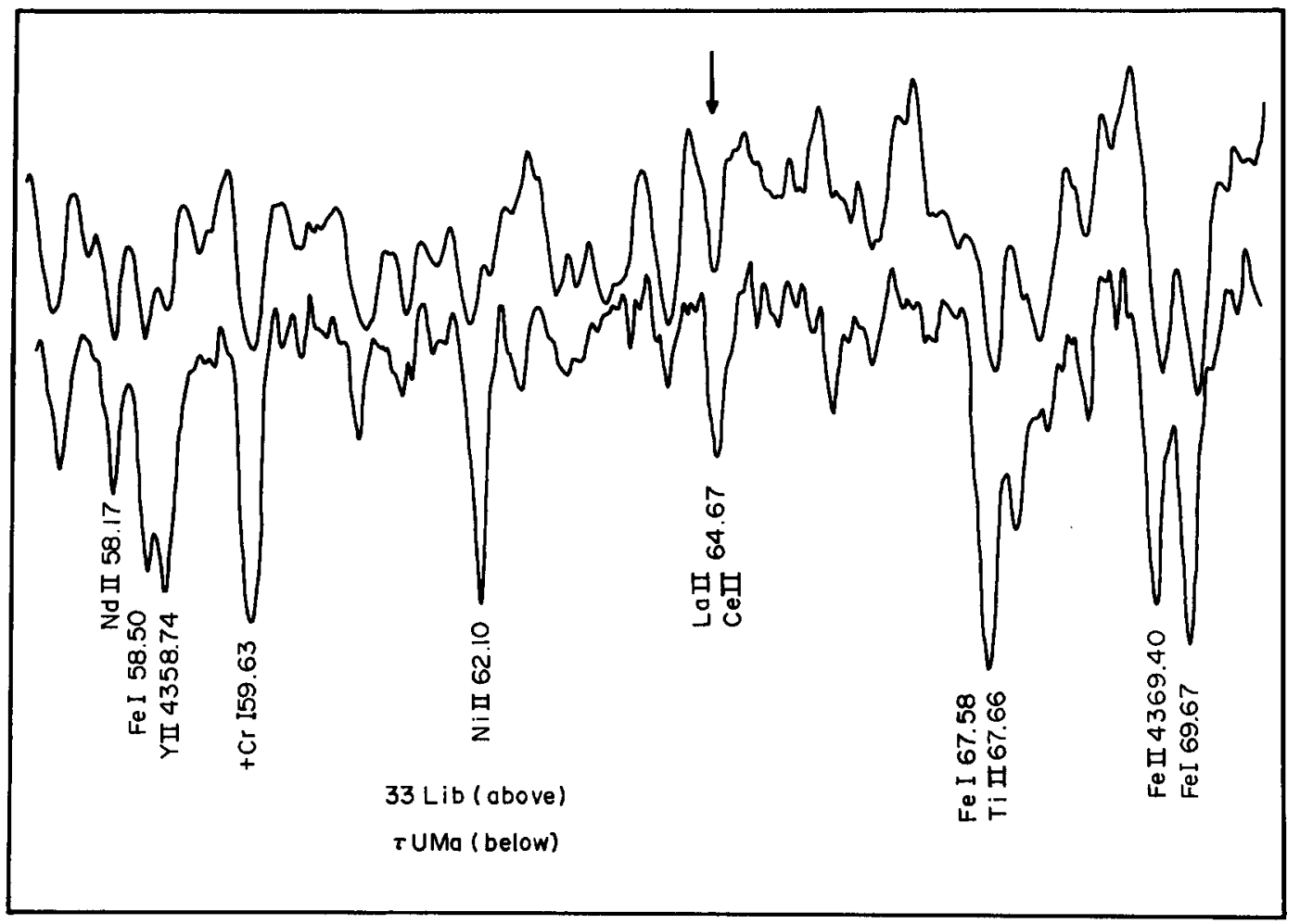

Figure 3. Same format as Figure 1 for the Ap star $33 \mathrm{Lib}$ and the Am or Fm star $\tau$ UMa. Zero intensity for 33 Lib is not reliably determined on the tracing and is therefore not indicated on the figure.

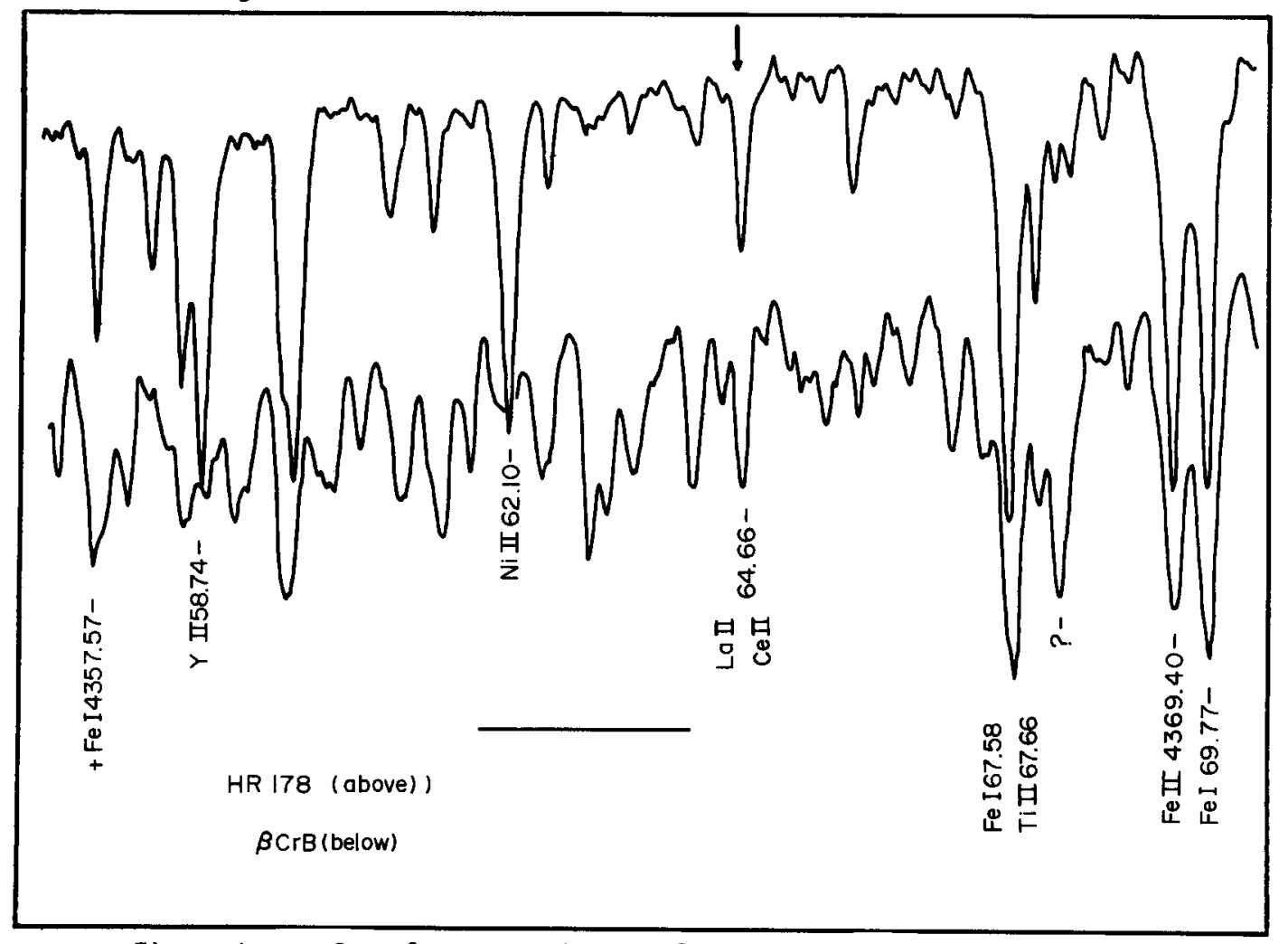

Figure 4. Same format as Figure 1 for the Am star HR 178 and the Ap star $\beta \mathrm{CrB}$. Note the weakness of $\mathrm{Nd} \mathrm{II,Y} \mathrm{II} \mathrm{and} \mathrm{Ni} \mathrm{II} \mathrm{in} \beta$ $\mathrm{Cr} B$, which has, generally, a richer line spectrum. 
La II. There is no indication that this line is systematically stronger in the Ap stars. Some idea of the relative level of ionization in these stars may be obtained by comparing Fe II $\lambda 4369.40$ with Fe I $\lambda 4369.77$. This line ratio implies that the level of ionization is comparable in all of these stars; if there is a difference, the Fe II/Fe I ratio may be greater in the Am stars implying, cet. par., that the Ce and/or La abundances would be somewhat higher in the Am's.

If the strengths of intermediate and weak lines in these Ap and Am stars are comparable and the level or ionization is the same, there is no way that conventional analyses of these spectra should yield abundances of Ce or La which differ by one or two orders of magnitude! Only if the continuous opacity in the atmosphere of the Am stars were one to two orders of magnitude lower than in the Ap's could this be maintained. Such an opacity difference would have bizarre effects on the strengths of other (eg. iron group) lines, which are not observed We do believe that the atmospheres of the Am and Ap stars have certain anomalies (see below) but they are not capable of rationalizing the traditional views about abundance ratios of rare earths in these two varieties of chemically peculiar stars.

We must make some attempt to understand why mistaken notions about the rare earths have been held for so long. There are many reasons. First, there are some Ap stars in which the rare-earth abundances are undoubtedly several orders of magnitude higher than in the group whose spectra are illustrated in Figures 1-4. One has only to think of Przybylski's star (HD 101065), which is certainly a c001 (Ap?) star. Second, stellar spectroscopists attention probably focused on the Eu II lines, which are broadened by hyperfine structure, and are intrinsically stronger than the Ce II and La II lines because of the simple term structure of Eu II. There is evidence that the Eu II lines are typically stronger in the Ap stars than the Am's. We would not deny that some of the rare earth elements typically have larger overabundance factors in the Ap stars. Europium may be one such element. One must also keep in mind that the relative abundances of the lanthanides are not the same from one Ap star to another. We have called attention at various times to the unusual strength of $\mathrm{Sm}$ II and the weakness of $\mathrm{Nd}$ II in $10 \mathrm{Aql}$ (cf. $\gamma$ Equ) which is illustrated in Figure 2 (cf. Nd II $\lambda 4358.17$ ). The spectrum of $\beta$ CrB shows meager evidence for either Nd II or Sm II, while La II, Ce II, Eu II and Gd II are strong. We have referred to this as a neodymium-samarium "hole." Both Nd II and Sm II are weaker in the Ap star B CrB than in the Am stars 32 Aqr etc.

But the strongest reason why abundance studies or rare earths in cool An and Ap stars have been so different is that they were done by different people. It has been traditional for abundance workers to study either the Ap's or the Am's. It is to be expected that systematic errors will arise between different workers, and we must keep in mind that a great deal of attention was not devoted to lanthanide spectra. Often abundances rested on a few, 
insecurely identified lines.

\section{I. SYNTHESIS}

If the overabundance factors of the lanthanides in typical magnetic Ap stars resemble those in the Am's, we may use Hundt's (1972) exemplary study of 63 Tau to obtain quantitative estimates for both kinds of star. Hundt compared the solar lanthanide abundance of Grevesse and Blanquet (1969) with his 63 Tau values, obtaining abundance excesses between 1.5 and 2.0 dex for La, Ce, Pr, Nd, and Sm. In both studies Corliss and Bozman's (1962) $10 \mathrm{~g}$ gf's were used, so the differential abundances should be reasonably good. We have adjusted Hundt's absolute abundances from 6 La II lines with the help of new gf values based on lifetime measurements of Andersen et a1. (1975) and Arnesen et a1. (1977), and obtain an excess of 2.1 dex.

It may be that typical Am lanthanide excesses are slightly larger than the factor of 10-30 estimated, for example, by Hack (1976), while those in typical coolest Ap stars are at the lower extreme of her range $10^{2}-10^{3}$. Her estimates are reasonable since the term "cool Ap stars", as used for example by Adelman (1973), included stars hot enough to have ionization corrections of at least an order of magnitude. In the silicon stars, with effective temperatures above $10,000^{\circ} \mathrm{K}$, the ionization corrections can reach nearly four orders of magnitude.

No stars in the non-magnetic sequence have the large abundance excesses 3-4 dex typical of those silicon stars showing the presence of lanthanides. Indeed, in the manganese stars, there is very little evidence for the presence of lanthanides, although it is easy to show that overabundance factors of 2-3 dex could escape detection (Cowley 1976).

If we omit from consideration those extreme Ap stars such as Przybylski's star (HD 101065) or HR 465, we find a more or less steady increase in rare earth abundances in magnetic Ap stars that closely follows the supposed ionization. If abundances of lanthanides in cool magnetic and non-magnetic Ap stars are similar, as we discussed in Section II, what causes an increase with temperature in the magnetic sequence only? Phenomenologically, what we find is that the strengths of the lines of the second spectra of the lanthanides do not fall off as rapidly as the Saha Equation would predict for constant abundances. Since the abundances are similar in those stars where ionization is not important there is a likelihood that an error is somehow being made in the traditional LTE models using the Saha Equation.

Nothing can be proved at this time. We can only suggest to the stellar-atmosphere theoreticians that this question deserves attention. While we have suggested an explanation in terms of non-LTE or stellar atmospheres, the same observations may comport well with diffusion models (Michaud 1977). Efforts, extending the work of Stepien (1978) to specific line 
spectra may prove fruitful. This remains to be seen.

\section{ANOMALOUS Am ATMOSPHERES}

Anyone who has classified spectra knows that Am stars resemble (sub) giants. Near FO, the differences between a late Am and an FO IV are extremely subtle, and often lead to discrepancies in published types. In the early 1960's extensive work by Conti (1965) and Van't Veer Menneret (1963) showed that the peculiar abundances in Am stars could not be explained away by the introduction of an anomalous atmosphere.

An excellent historical review of the problems of interpretation of Am stars is given by Böhm-Vitense (1976). Almost all efforts on these objects have been devoted to determining abundance anomalies using more or less standard models or attempting to explain away all abundance anomalies with the aid of some special mechanism. Surprisingly little attention has been devoted to peculiar abundances in peculiar atmospheres.

Böhm-Vitense (1960) pointed out that the central intensity of the K line of Ca II was markedly deeper in the Am stars 63 Tau and 16 Ori than in the Fo $V$ stars $\gamma$ Vir $N$ and $\gamma$ Vir S. Hundt (1972) allowed for a lowering of the surface temperature of 63 Tau by $600^{\circ} \mathrm{K}$ which he attributed to line blanketing. This lowering of the boundary temperature is a strictly LTE effect, and since the cores of the lines are formed high in the atmospheres of these stars, the LTE assumption is obviously open to question.

Whatever the proper interpretation may be, it is clear that the line depths in cool Am stars are greater than in either normal dwarfs or the magnetic Ap's (see Figures 1-4) at the same temperature. This appears to be generally true for most strong lines. The reader who wishes to see further examples may consult the illustrations in Cowley et al. (1977), where the region near $\lambda 3859$ is shown for the stars of Figures 1-4 except for HR 178 and 33 Lib. The general effect is striking.

In most of the spectra that we have examined, the line depths are controlled by stellar rotation. However, it is quite impossible to maintain that the values of $v$ sin $i$ are higher in those Ap stars shown in Figures 1-4 than in the Am's. It is the Ap's which have the sharper spectral lines, yet their line depths are less.

The sharpest-1ined Am spectrum of our experience is that of HR 178. We wish to thank Dr. Van't Veer Menneret for calling this spectrum to our attention. The line depths in this star exceed those in the F8V star $U$ And, while the $H \gamma$ profile indicates that HR 178 is slightly hotter than the F2V standard $\sigma$ Boo. It is, of course, known that line depths generally increase in the spectra of later stars.

We must mention in this connection the conclusion reached by Greenstein (1947) in his classical study "Spectrophotometry of the F stars and of $\tau$ Ursa Majors". Greenstein con- 
cluded that the central line depths in $\tau$ UMa were similar to those in the other $F$ stars of his study. These other F stars, however, were not dwarfs. They were $\alpha$ Per (F5 Ib), $\rho$ Pup (F6 II), $\theta$ UMa (F6 III), and a CMi (F5 IV). These stars do indeed have deeper lines than early F dwarfs, so Greenstein's observations are in good accord with our own.

Deep spectral lines suggest a "shell" to an observational spectroscopist. of course, we know that Am stars are not shell stars. However, these deep line cores may indicate some kind of an extended upper atmosphere of the kinds suggested by Böhm-Vitense (1960) and investigated by Conti (1965) and Van't Veer Menneret (1963). We believe that such models might be considered afresh, not as a means of explaining away all abundance anomalies, but as a means of accounting for the observed line profiles.

Two rather recent papers by Abt and Morgan (1976) and Hardorp (1977) discuss the question of Am star phenomenology from the point of view of low dispersion spectroscopy. The resemblance of certain Am features to those of higher luminosity stars is well illustrated in the former paper, which includes a statement by Dr. Dimitri Mihalas suggesting the possible relevance of Böhm-Vitense's model with distended or "blown up" outer layers. Hardorp criticized certain aspects of this paper, quite correctly pointing out that if the Am stars have abundance anomalies, certain standard luminosity criteria cannot be safely used. He mentioned specifically that the ratio ( $\mathrm{Sr}$ II $\lambda 4077 / \mathrm{Fe}$ I $\lambda 4046$ ) can be expected to yield a higher luminosity in a star with an enhanced strontium abundance, which the Am's surely have.

Some of the other features found by Abt and Morgan, Hardorp found more difficult to explain in terms of abundances, but he pointed out that careful model atmosphere analyses ought to reveal atmospheric peculiarities if they exist and that abundance analyses (eg. Smith 1973) did not need to envoke distended atmospheres in addition to chemical peculiarities. In fact, Bolton (1970) had shown by spectrum synthesis how abundance anomalies could influence other MK criteria. However, neither his work nor highly revelant remarks by Conti (1965) were discussed in these recent papers.

We quote from Conti's paper: "The statement that somewhat decreased-gravity mode1 atmospheres fit the observations of these Am stars again supports the statements that the electron pressure in Am stars resembles that of giants and supergiants rather than dwarfs." Conti goes on to emphasize that these models do not explain away the abundance anomalies. Much of Hardorp's criticism of the Abt-Morgan paper must be seen in this light: the peculiar abundances appear to him to be more firmly established than the low gravity atmospheres. Bolton found that some of the MK luminosity criteria are particularly sensitive to microturbulence because the eye picks up blends of saturated lines. It is well known that the maximum line depth parameter, Unsöld's (1955) $\mathrm{R}_{c}$ influences equivalent widths in much the same way as microturbulence. Indeed, these two variables are the major factors influencing 
the strengths of saturated lines. It is, of course, precisely this $R_{c}$ which our observations, as well as those of Greenstein (1947), tell us are larger in Am stars than dwarfs with the same effective temperature.

An LTE treatment of the $R_{c}$ parameter is quite unrealistic. Even though enhanced line blanketing in An stars will surely improve the agreement between theory and observation with

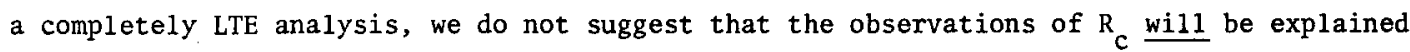
in this way. If distended outer atmospheres are of relevance, it is quite possible that a scattering mechanism may play a strong role in the formation of these deep line cores.

We leave these problems for the theoreticians, pointing out that the observations require investigations of both line formation and model (upper) atmospheres.

\section{THE STRANGE BEHAVIOR OF YTTRIUM}

Yttrium $(z=39)$ is often considered to be a rare earth, although it is in the fifth period of the Periodic Table rather than the sixth, where the lanthanides are found. Its first three ionization energies are closely similar to those of the lanthanides.

There is a marked contrast in the overall behavior of this element in the magnetic and non-magnetic sequences of chemically peculiar stars. Y II is usually well identified among the coolest stars of both sequences, but among the hotter stars, Y II lines show a definite tendency to be stronger in the non-magnetic objects, the mercury-manganese stars. Often yttrium is weak or absent in the spectra of magnetic Ap stars that are rich in lanthanides. Yttrium abundances show a high degree of correlation with stellar effective temperature (Allen 1977). As with the lanthanides, it is useful to ask if this correlation results from errors in our analysis.

The theoretical problem posed by these observations is challenging. Any under-ionization, for example, which enables second lanthanide spectra to persist in the hotter magnetic Ap stars is likely to apply also to yttrium.

Yet yttrium definitely favors the non-magnetic sequence. Why? We can only suggest that the intrinsic photospheric abundances of yttrium are higher in the hotter non-magnetic stars for a reason that must be closely connected with the overall question of peculiar abundances in upper main sequence stars.

\section{CONCLUDING REMARKS}

High resolution spectra of late $B, A$, and early F stars show an enormous variety which is imperfectly understood. For many modern astronomers, the only important question has been whether nuclear processes have played a role in the creation of these anomalies, or if the non-nuclear theories will account for all of these peculiar spectra. Yet both contingen- 
cies hold promise of opening fascinating areas for future research.

The possible relevance of nuclear mechanisms requires us to consider new domains in stellar evolution and nucleosynthesis. On the other hand, if the non-nuclear mechanisms prove adequate, we may look upon the surfaces of these stars in much the same way that the geologist views the chemically varigated crust of the earth and planets. From an examination of the composition of surfical rocks, the geochemist can infer much about the age and history of the materials. If such interesting questions can be investigated with the earth's surface chemistry, we can do similar things with the stars.

It is a pleasure to acknowledge once again my indebtedness to the Director and Staff of the Dominion Astrophysical observatory. I am grateful to Drs. D. S. Leckrone and F. Praderie for their comments on an early version of this paper. This research was supported by the National Science Foundation. 


\section{REFERENCES}

Abt, H., and Morgan, W. W. 1976, Ap. J. 205, 446.

Adelman, S. J. 1973, Ap. J., 183, 95.

Adelman, S. J. 1974, Ap. J. Supp1., 28, 51.

Aikman, G. C. L., Cowley, C. R. and Crosswhite, H. M. 1979, Ap. J., 232, 812.

A11en, M. S. 1977, Ap. J., 213, 121.

Arnesen, A., Bengtsson, A., Hallin, R., Lindskog, J., Nordling, C., and Nordland, T. 1977, Phys. Scr., 16, 31 .

Andersen, T., Poulsen, 0., Ramanujam, P. S., and Petkov, A. P. 1975, Solar Phys., 44, 257.

Böhm-Vitense, E. 1960, Zs. f. Ap., 49, 243.

Böhm-Vitense, E. 1976, in Physics of Ap Stars, I.A.U. Colloquium No. 32, ed. W. W. Weiss (Vienna: Finsterle and Co.) p. 633.

Bolton, C. T. 1970, Astron. Ap., 14, 233.

Conti, P. S. 1965, Ap. J. Supp1., 11, 47.

Corliss, C. H., and Bozman, W. R. 1962, N.B.S. Monog., 53.

Cowley, C. R. 1976, in Physics of Ap Stars, I.A.U. Colloquium No. 32, ed. W. W. Weiss (Vienna: Finsterle and Co.) p. 275.

Cowley, C. R. 1979, Mem. Soc. Roy. Liege, 12, 373 (Proc. 22nd Internat. Ap. Symposium Leige).

Cowley, C. R., Aikman, G. C. L., and Fisher, W. A. 1977, Pub. Dom. Ap. Obs., 15, 37.

Cowley, C. R., and Aikman, G. C. L. 1980, Ap. J., in press.

Deutsch, A. 1956, in Vistas in Astronomy, 2, 1421.

Greenstein, J. L. 1947, Ap. J., 107, 151.

Grevesse, N., and Blanquet, G. 1969, Solar Phys., 8, 5.

Hack, M. 1976, in Physics of Ap Stars, I.A.U. Colloquium No. 32, ed W. W. Weiss (Vienna: Finsterle and Co.) p. 255.

Hardorp, J. 1977, Ap. J., 214, 408.

Hartoog, M. R., Cowley, C. R., and Cowley, A. P. 1973, Ap. J., 182, 847.

Hensberge, H., Cowley, C. R., van Rensbergen, W., and Aikman, G. C. L. 1980, Astron. and Ap., in press.

Hundt, E. 1972, Astron. Ap., 21, 413.

Kurucz, R. L. 1979, private communication.

Leckrone, D. S. 1980, in Highlights of Astronomy, vol. 5, in press.

Michaud, G. 1977, in Highlights of Astronomy, Vo1. 4, Part II, ed. E. A. Müller (Dordrecht: D. Reidel) p. 177 .

Smith, M. A. 1973, Ap. J. Suppl., 25, 277.

Stepien, K. 1978, Astron. and Ap., 70, 509.

Unsöld, A. 1955, Physik der Sternatmospharen, (2nd ed.; Ber1in: Springer Verlag) see p. 413.

Van't Veer-Menneret, C. 1963, Ann. d'Ap., 26, 289.

Worre11, G., and Wilson, A. M. 1972, Nature, 236, 15. 